\title{
DEVELOPMENT OF A ZEBRAFISH LARVAE INFECTION MODEL TO STUDY VIRULENCE FACTORS OF A. hydrophila
}

\author{
Alejandro Romero ${ }^{1}$, Paolo Roberto Saraceni ${ }^{1}$, Patricia Pereiro ${ }^{1}$, Juan Tomás ${ }^{2}$, Susana Merino ${ }^{2}$, Antonio \\ Figueras $^{1}$, Beatriz Novoa ${ }^{1 \S}$
}

1 Instituto de Investigaciones Marinas (IIM), CSIC, Eduardo Cabello 6, 36208 Vigo, Spain.

2. Department of Microbiology, University of Barcelona, Diagonal 643, Barcelona 08071, Spain.

\begin{abstract}
Aeromonas hydrophila is a Gram negative bacteria widely distributed in aquatic environments. It is an opportunistic pathogen for fish and terrestrial animals including mammals. In fish it causes motile hemorrhagic septicemia leading to a high mortality and economic losses in aquaculture. In humans it is an emerging pathogen provoking gastroenteritis, septicemia, and skin and soft tissue infections. Some clinical conditions like cancer, hepatic diseases, diabetes and trauma increase the risk to develop a fatal A. hydrophila infection. The pathogenicity of A. hydrophila is multifactorial depending on several virulence factors, including surface polysaccharides (capsule, lipopolysaccharide, and glucan), S-layers, iron-binding systems, exotoxins and extracellular enzymes, secretion systems, fimbriae and other nonfilamentous adhesins, motility and flagella
\end{abstract}

In this work two different models of experimental infection have been developed in zebrafish larvae (Danio rerio) to study the pathogenicity of $A$. hydrophila: microinjection and bath immersion. The transparency of zebrafish larvae and the availability of transgenic fish lines showing fluorescent immune cells or immune marker genes allow the evaluation of the inflammatory processes in vivo and the analysis of the host-microbe interactions.

The infection model by bath immersion of injured zebrafish larvae is an alternative non-mammal model to study the implication of different virulence factors in the pathogenicity of $A$. hydrophila. This new model mimics the natural route of infection by water and an additional injury in the fins provides a natural portal of entry to the bacteria.

The implication of the virulence factors in the pathogenesis of $A$. hydrophila was evaluated by experimental infections using the following specific mutant: AH-1::aopB lacking the T3SS, AH-1 $\Delta$ rmlB which is devoid of the O-antigen LPS with a complete LPS-core, the AH-1 $\triangle$ wahD which lacks the O-antigen LPS and part of the LPS outer-core, AH-1 $\Delta$ vapA, lacking the gene coding for the S-layer protein, a non-polar flagellated mutant AH-1 $\Delta$ FlaB-J unable to swim, and the AH-1::motX mutant, being non-motile but able to produce polar flagellum.

All the results highlight the importance of the correct selection of the infection model to analyse the fish immune response and the implication of the virulence factors of $A$. hydrophila.

\section{KEYWORDS}

Zebrafish, Aeromonas, infection model, virulence factors, immune response

§Corresponding author. Tel.: +34 986231930; Fax: +34 986292762.

E-mail address: virus@iim.csic.es 